\title{
Respuesta adaptativa del niño con síndrome de bajo gasto cardiaco a los cambios de posición en la unidad de cuidado intensivo pediátrico'
}

\author{
Judy Ximena Ramos Garzón² \\ Sandra Rocío Guáqueta Parada³
}

doi:10.11144/Javeriana.IE16-2.rans

Cómo citar: Ramos Garzón JX, Guáqueta Parada SR. Respuesta adaptativa del niño con síndrome de bajo gasto cardiaco a los cambios de posición en la unidad de cuidado intensivo pediátrico. Investig Enferm. Imagen Desarr. 2014;16(2):115-132. doi:10.11144/ Javeriana.IE16-2.rans

1. Artículo producto de la investigación realizada entre 2010 y 2011 en la unidad de cuidado intensivo pediátrico de una institución de tercer nivel en la ciudad de Bogotá, Colombia. Recibido: 18 de febrero de 2014. Revisado: 1 y 21 de abril de 2014. Aprobado: 23 de mayo de 2014. Artículo derivado del proyecto: Respuesta adaptativa en el modo fisiológico del lactante con bajo gasto cardiaco en estado critico, a las intervenciones cotidianas de enfermería en la UCIP, financiado por la Universidad Nacional de Colombia.

2. Magíster en Enfermería para el Cuidado de la Salud Cardiovascular, especialista en Cuidado Intensivo. Docente ocasional, Universidad Nacional de Colombia. Correo electrónico: ximenaramosgarzon@gmail.com

3. Magíster en Educación, especialista en Enfermería Cardiorrespiratoria. Profesora asociada, Universidad Nacional de Colombia. Correo electrónico: srguaquetap@unal.edu.co 


\section{Resumen}

Introducción: Roy, en su modelo de adaptación, plantea que un estímulo contextual como una intervención de enfermería genera en el sistema humano una respuesta adaptativa que puede ser efectiva o inefectiva. Objetivo: Describir y analizar la respuesta adaptativa en el modo fisiológico en lactantes de 2 a 23 meses de edad con bajo gasto cardiaco en estado crítico, al realizar intervenciones cotidianas de enfermería en la unidad de cuidado intensivo pediátrica. Método: Observacional-descriptivo de abordaje cuantitativo y diseño de mediciones repetidas. Los indicadores propuestos de adaptación fisiológica positiva que se midieron fueron: tensión arterial sistólica, frecuencia cardiaca y llenado capilar. La muestra $(n=75)$ la constituyeron niños lactantes y se dividió en tres grupos según la severidad del bajo gasto cardiaco $(\mathrm{n}=25)$. Análisis estadístico: Análisis no paramétrico en SPSS 15 con prueba de Wilcoxon, Kruskal-Wallis y chi cuadrado. Análisis gráfico con Boxplot. Resultado: La intervención de enfermería (cambio de posición) tiene un efecto prolongado y significativamente negativo $(p<0,05)$ en la capacidad adaptativa fisiológica de los lactantes en estado crítico; depende de la severidad del bajo gasto cardiaco: a mayor severidad del bajo gasto cardiaco, mayor presencia de adaptación inefectiva. Conclusiones: Es posible la cuantificación de la capacidad fisiológica de adaptación de un sistema humano a través de indicadores sensibles y específicos de adaptación fisiológica positiva. La técnica, duración y frecuencia de los cambios de posición del lactante con bajo gasto cardiaco en estado crítico deben ser una decisión de enfermería basada en la respuesta hemodinámica y las necesidades de cuidado de cada paciente.

Palabras clave: adaptación; lactante; gasto cardiaco bajo; atención de enfermeria; unidades de cuidado intensivo pediátrico

\section{Adaptive Response of Children with Low Cardiac Output Syndrome to Changes of Position in the Pediatric Intensive Care Unit}

\section{Abstract}

Introduction: Roy, in his adaptation model, suggests that a contextual stimulus as a nursing intervention generates in the human system an adaptive response that can be effective or ineffective. Objective: To describe and analyze the adaptive response in the physiological mode in infants 2-23 months of age with low cardiac output in critical condition, when performing daily nursing interventions in the pediatric intensive care unit. Method: Observational-descriptive quantitative approach and repeated measures design. The proposed indicators of positive physiological adaptation measured were: systolic blood pressure, heart rate and capillary refill. The sample (n $=75$ ) was made up of children and infants. It was divided into three groups according to the severity of low cardiac output $(n=25)$. Statistical analysis: nonparametric analysis in SPSS 15 Wilcoxon, Kruskal-Wallis and chi-square. Boxplot graph analysis. Result: The nursing intervention (change of position) has a long and significant negative effect $(\mathrm{p}<0.05)$ in the physiological adaptive capacity of infants in critical condition; It depends on the severity of low cardiac output: the greater severity of low cardiac output, the greater the presence of ineffective adaptation. Conclusions: It is possible to quantify the physiological adaptability of a human system through sensitive and specific indicators of positive physiological adaptation. The technique, duration and frequency of changes in position of the infant with low cardiac output in critically condition should be a nursing decision based on hemodynamic response and care needs of each patient.

Keywords: adaptation; infant; low cardiac output; nursing care; pediatric intensive care units 


\section{Resposta adaptativa da criança com sindrome de baixo débito cardiaco às mudanças de posição na unidade de terapia intensiva pediátrica}

\section{Resumo}

Introdução: Roy, no seu modelo de adaptação, sugere que um estímulo contextual tal como uma intervenção de enfermagem gera no sistema humano uma resposta adaptativa que pode ser eficaz ou ineficaz. Objetivo: Descrever e analisar a resposta adaptativa no modo fisiológico em lactentes de 2 para 23 meses de idade com baixo débito cardíaco em estado crítico, ao realizar intervenções cotidianas de enfermagem na unidade de terapia intensiva pediátrica. Método: Observacional-descritivo de abordagem quantitativa e desenho de medições repetidas. Os indicadores propostos de adaptação fisiológica positiva medidos foram: tensão arterial sistólica, frequência cardiaca e enchimento capilar. A amostra $(n=75)$ foi constituída por crianças lactentes e dividiu-se em três grupos segundo severidade do baixo débito cardiaco ( $\mathrm{n}=25)$. Análise estadistica: Análise não paramétrica em SPSS 15 com proba de Wilcoxon, Kruskal-Wallis e qui-quadrado. Análise gráfico com Boxplot. Resultado: A intervenção de enfermagem (mudança de posição) tem um efeito prolongado e significativamente negativo $(\mathrm{p}<0,05)$ na capacidade adaptativa fisiológica dos lactentes em estado crítico; depende da severidade do baixo débito cardíaco: para maior severidade do baixo débito cardiaco, maior presença de adaptação ineficaz. Conclusões: Quantificação da capacidade fisiológica de adaptação de um sistema humano através de indicadores sensiveis e específicos de adaptação fisiológica positiva é possivel. A técnica, duração e frequência das mudanças de posição do lactente com baixo débito cardíaco em estado crítico devem ser decisões de enfermagem baseadas na resposta hemodinâmica e as necessidades de cuido de cada paciente.

Palavras chave: adaptação; lactente; débito cardíaco baixo; atendimento de enfermagem; unidades de terapia intensiva pediátrica 


\section{Introducción}

El síndrome de bajo gasto cardiaco se reconoce como una condición clínica caracterizada por la incapacidad del corazón para mantener una perfusión adecuada de los órganos y tejidos corporales (1). Es una entidad frecuente en la unidad de cuidado intensivo pediátrico y constituye un punto convergente al que se puede llegar por diferentes vías o estados patológicos, como los defectos congénitos del corazón con falla cardiaca, las enfermedades respiratorias, la hipertensión pulmonar, la cirugía cardiaca, la sepsis, el trauma, entre otras.

Las cardiopatías congénitas son la principal causa primaria de bajo gasto cardiaco en pediatría. Las cardiopatías que más se le asocian son la conexión anómala total de venas pulmonares obstructivas, la trasposición de grandes arterias y el síndrome del ventrículo izquierdo hipoplásico (2). Una generalidad de los pacientes con bajo gasto cardiaco, independiente de su origen, es la inestabilidad hemodinámica, situación que agrega gran complejidad al cuidado de enfermería del paciente en estado crítico.

Las intervenciones de enfermería deben responder a las necesidades de cuidado específicas para estos pacientes, manteniendo la estabilidad y preservando la vida, lo que constituye un verdadero reto en el momento de planear intervenciones de cuidado eficientes, pero seguras para las personas. Según la teoría de adaptación de Callista Roy, en el modo fisiológico la capacidad de adaptación puede estimarse a partir de indicadores positivos (3). Para esta investigación (4), dado que la población objeto son lactantes con bajo gasto cardiaco, fue necesario construir nuevos indicadores específicos de adaptación tomando como base el modelo de adaptación de Roy y los parámetros fisiológicos que tienen utilidad en el reconocimiento y evaluación del síndrome de bajo gasto cardiaco en pediatría.

En general, el paciente lactante tiene menor capacidad de respuesta compensatoria efectiva ante la inestabilidad hemodinámica por bajo gasto cardiaco, dadas sus características fisiológicas particulares, como la inmadurez biológica, la reserva funcional restringida del corazón joven, el elevado nivel basal de actividad simpática y la pobre respuesta a catecolaminas, por una densidad disminuida de los betaadrenorreceptores (5). Es indispensable para la enfermera conocer la respuesta adaptativa en el modo fisiológico del paciente pediátrico en estado crítico para comprender y predecir con mayor precisión el efecto de las intervenciones de cuidado que planee para este grupo de pacientes.

En el modelo de adaptación de Callista Roy se puede identificar que un estímulo focal (como es el bajo gasto cardiaco) y un estímulo contextual (como la intervención de enfermería cambio de posición) influyen en el subsistema regulador del niño lactante y generan una respuesta adaptativa del modo fisiológico, que se puede medir con indicadores de adaptación positiva como aparece en la figura 1. 


\section{MODELO DE ADAPTACIÓN DE CALLISTA ROY}

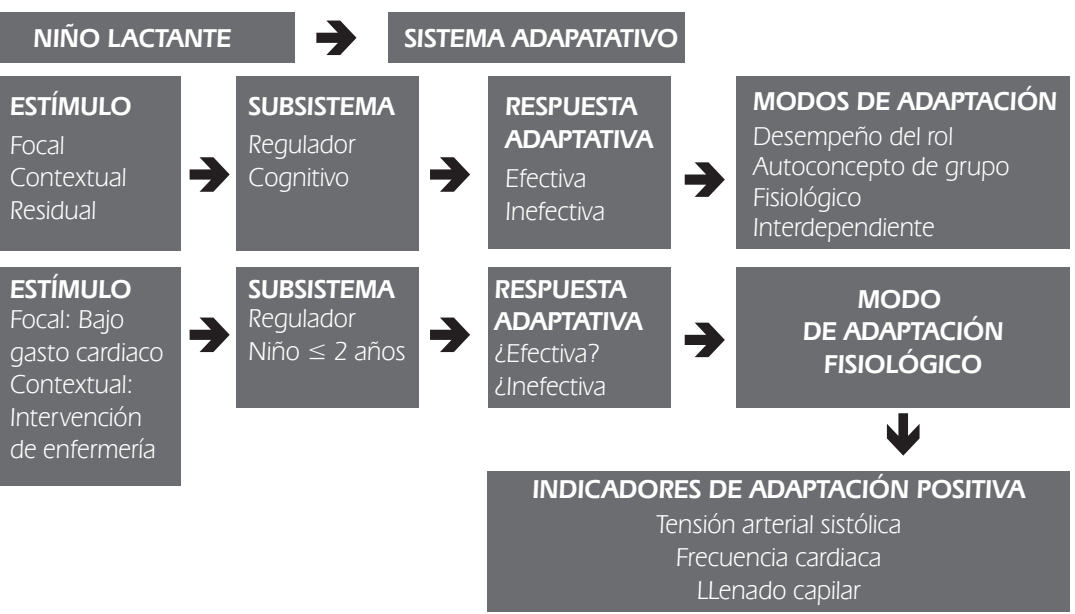

Figura 1. Mapa teórico de la investigación

Fuente: tesis de maestría Respuesta adaptativa en el modo fisiológico del lactante con bajo gasto cardiaco en estado crítico, a las intervenciones cotidianas de enfermería en la unidad de cuidado intensivo pediátrico.

Con el tiempo y las investigaciones realizadas, Roy ha venido complementando y enriqueciendo su modelo conceptual hasta llegar a una visión filosófica de reciprocidad o interactiva-integrativa, la cual concede a la enfermera una mayor participación, en cuanto está en capacidad de producir estímulos positivos que favorezcan la adaptación (6).

\section{Indicadores de adaptación positiva}

Con base en los postulados teóricos del modelo de adaptación de Roy y en los parámetros fisiológicos que tienen utilidad en el reconocimiento y evaluación del síndrome de bajo gasto cardiaco en pediatría, a continuación se definen los indicadores de adaptación positiva construidos para esta investigación con el objetivo de medir la respuesta adaptativa en el modo fisiológico, antes de realizar la intervención de enfermería: cambio de posición y después de esta.

Los signos clínicos que permiten identificar el bajo gasto cardiaco son: disminución en la intensidad del pulso, prolongación del tiempo de llenado capilar, piel fría y marmórea, aumento de la frecuencia cardiaca, tensión arterial sistémica disminuida o normal, oliguria o anuria (7). Para esta investigación se tomaron tres signos de bajo gasto cardiaco: tensión arterial sistólica, por tener mayor valor clínico en pediatría; frecuencia 
cardiaca y llenado capilar, este último como criterio de valoración general de la perfusión de la piel. El gasto urinario se excluye en este estudio, dado que las mediciones se realizan en intervalos muy cortos de tiempo (minutos) y algunos pacientes no tienen sonda vesical.

A continuación, como se muestra en las tablas siguientes, se establecieron los rangos de respuesta adaptativa efectiva (rango de valores normales para la edad) e inefectiva (por exceso o por defecto) según la literatura disponible para cada uno de los indicadores seleccionados para esta investigación: tensión arterial sistólica (tabla 1), frecuencia cardiaca (tabla 2) y llenado capilar (tabla 3 ).

TABLA 1. Indicador de adaptación positiva basado en la tensión arterial sistólica

\begin{tabular}{|l|c|c|c|}
\hline $\begin{array}{c}\text { Edad en } \\
\text { meses }\end{array}$ & $\begin{array}{c}\text { Respuesta } \\
\text { inefectiva: } \\
\text { hipotensión } \\
\text { (mm Hg) }\end{array}$ & $\begin{array}{c}\text { Respuesta efectiva: } \\
\text { concentración } \\
\text { afectada } \\
\text { (mm Hg) }\end{array}$ & $\begin{array}{c}\text { Respuesta inefectiva: } \\
\text { hipertensión } \\
\text { (mm Hg) }\end{array}$ \\
\hline $2-6$ & $\leq 71$ & $72-98$ & $\geq 99$ \\
\hline $7-12$ & $\leq 75$ & $76-105$ & $\geq 106$ \\
\hline $13-18$ & $\leq 82$ & $83-114$ & $\geq 118$ \\
\hline $19-23$ & $\leq 84$ & $85-117$ & $\geq 15$ \\
\hline
\end{tabular}

Fuente: basada en Shilkofski R. Cardiología. En: Manual Harriet Lane de pediatría. 17th ed. 2006. Horan M et al. Pediatrics 1987; 79(1):1-25. The fourth report on the Diagnosis, evaluation, and treatment of high blood pressure in children and adolescents. Pediatrics. 2004;114: 555-76.

TABLA 2. Indicador de adaptación positiva basado en la frecuencia cardiaca

\begin{tabular}{|c|c|c|c|}
\hline $\begin{array}{c}\text { Edad en } \\
\text { meses }\end{array}$ & $\begin{array}{c}\text { Respuesta } \\
\text { inefectiva: } \\
\text { bradicardia (LMP) }\end{array}$ & $\begin{array}{c}\text { Respuesta efectiva: } \\
\text { concentración } \\
\text { afectada (LPM) }\end{array}$ & $\begin{array}{c}\text { Respuesta } \\
\text { inefectiva: } \\
\text { taquicardia } \\
\text { (LPM) }\end{array}$ \\
\hline $2-6$ & $\leq 109$ & $110-175$ & $\geq 176$ \\
\hline $7-12$ & $\leq 104$ & $105-170$ & $\geq 171$ \\
\hline $13-18$ & $\leq 99$ & $100-160$ & $\geq 151$ \\
\hline $19-23$ & $\leq 89$ & $90-150$ & $\geq 161$ \\
\hline
\end{tabular}

BASADo en: American Heart Association. Soporte vital avanzado pediátrico: libro para el proveedor. Barcelona: Prous Science; 2008, p. 16. Craig J, 
TABLA 3. Indicador de adaptación positiva basado en el llenado capilar

\begin{tabular}{|c|c|}
\hline $\begin{array}{c}\text { Respuesta efectiva } \\
\text { (nivel comprometido) }\end{array}$ & \multicolumn{1}{c|}{$\begin{array}{c}\text { Respuesta inefectiva } \\
\text { hipoperfusión }\end{array}$} \\
\hline$\leq 2,99$ segundos & $\geq 3$ segundos \\
\hline
\end{tabular}

Basado En: American Heart Association. Soporte vital avanzado pediátrico: libro para el proveedor. Barcelona: Prous Science; 2008, p. 19.

Fue necesario establecer para los lactantes (niños y niñas de 2 a 23 meses) subgrupos de edad (2-6, 7-12, 13-18 y 19-23 meses), debido a la diferencia del valor normal para la edad como se evidencia en las tablas 1 y 2 de los indicadores tensión arterial sistólica y frecuencia cardiaca.

\section{Metodología}

Estudio observacional descriptivo con abordaje cuantitativo, realizado en la unidad de cuidado intensivo pediátrico de una institución de tercer nivel de atención en la ciudad de Bogotá (Colombia).

El propósito de un diseño descriptivo es analizar las relaciones observadas entre dos o más variables (8), más que inferir relaciones de causa-efecto (9). El objetivo de este estudio se centra en describir y analizar el efecto de las variables independientes (estímulo contextual: bajo gasto cardiaco y estímulo focal: cambio de posición) sobre las variables dependientes (frecuencia cardiaca, tensión arterial y llenado capilar), más que en establecer una relación causal absoluta.

La muestra se calculó por muestreo piloto empleando la ecuación de Schwerman; de esta manera, se incluyeron 75 lactantes entre los 2 y los 23 meses de edad, distribuidos en tres grupos $(n=25)$, según la severidad del bajo gasto cardiaco. Dicha severidad se estableció por la cantidad de inotrópicos y vasoactivos que recibía el paciente en infusión continua y a dosis terapéutica. Grupo I: un medicamento; grupo II: de dos a tres, y grupo III: cuatro o más.

Se implementó la metodología de mediciones repetidas de la siguiente manera: una medición inicial en el minuto cero (momento que antecede el inicio de la intervención de enfermeria) de las tres variables dependientes (tensión arterial sistólica, frecuencia cardiaca y llenado capilar), e inmediatamente se da inicio a la intervención de enfermería cambio de posición. Una vez finalizada la intervención, se registra el tiempo de duración y 5 minutos después se realiza la segunda medición, una tercera a los 15 minutos y la cuarta y última al completar 30 minutos. Con el fin de determinar la variabilidad de los indicadores y la adaptación fisiológica de los lactantes.

\section{Criterios de inclusión}

1. Lactante con edad entre 45 dias y 24 meses.

2. Lactante con bajo gasto cardiaco que recibe infusión intravenosa continua de al menos un inotrópico o vasoactivo en dosis terapéutica. 
3. Lactante con monitoreo continuo de la tensión arterial y la frecuencia cardiaca.

\section{Criterios de exclusión}

Paciente que, cumpliendo con los criterios de inclusión en el momento de la medición inicial, se encuentre en fase de adaptación inefectiva, es decir, en la franja del indicador que equivale a signos vitales inapropiados para la edad, ya sea por exceso o por defecto.

\section{Criterios para suspender la intervención}

Fue deber ético del investigador informar la presencia de un cambio abrupto en los signos vitales del paciente durante la intervención de enfermería o en las mediciones posteriores que se realicen. Se suspendió la secuencia de mediciones repetidas cada vez que el paciente requirió una intervención no postergable o urgente, ya sea de enfermería, terapia, pediatría o cualquiera de los integrantes del equipo de la unidad de cuidado intensivo pediátrico.

\section{Consideraciones éticas}

Consideración y cumplimiento de los principios éticos $(10,11)$ y las disposiciones legales vigentes (12) en esta investigación: veracidad, de las mediciones y de los datos y los resultados obtenidos. Reciprocidad, a través de la socialización de los resultados de la investigación con la comunidad interesada y los padres de los niños participantes. Respeto por la autonomía, validado por medio del derecho a la autoderminación y el derecho al conocimiento de la información, con la aplicación del consentimiento informado. No maleficencia, al elegir para esta investigación un diseño no experimental dado el estado de vulnerabilidad de los sujetos y la inclusión de los criterios de suspensión de las mediciones repetidas y el aviso inmediato por parte del investigador al médico tratante, en caso de observar cambios drásticos en el estado hemodinámico del niño. Beneficencia, porque con esta investigación se pretende obtener información objetiva y confiable que contribuya al desarrollo del conocimiento disciplinar y de la práctica clínica, a fin de optimizar la atención de enfermería de los niños en estado crítico. Justicia, trato imparcial de todos los sujetos y distribución equitativa de riesgos y beneficios al compartir igualdad de condiciones.

\section{Limitaciones}

Esta investigación, como toda que se realice en una unidad de cuidado intensivo, estuvo limitada - como es lógico- por la criticidad de los pacien-

122 tes y por su número. Por esa razón se recurrió a un muestreo piloto para calcular la muestra $(\mathrm{n}=75)$, la cual es significativa si se tiene en cuenta 
la especificidad del diagnóstico (bajo gasto cardiaco con requerimiento de inotrópicos o vasoactivos) y la escasa cantidad de camas de unidad de cuidado intensivo pediátrico. Adicionalmente, en la ecuación de Schwerman empleada para el cálculo del tamaño muestral se determinó un error a del $5 \%$ el cual es "clínicamente significativo" (13).

\section{Análisis estadístico}

Este estudio es observacional descriptivo, porque de ninguna manera podría ser experimental o semiexperimental, dadas las implicaciones éticas para este grupo de pacientes. Esto no quiere decir que la exploración estadística deba ser limitada. Para el análisis de los datos se empleó la versión 15 del Stadistical Package for the Social Sciences (SPSS). Inicialmente se aplicó la prueba de normalidad de Shapiro-Wilk y se encontró que cerca del $40 \%$ de las variables no tenía una distribución normal de los datos, situación atribuible a la gran variabilidad de los signos vitales en los lactantes, por lo que se realiza un análisis estadístico con pruebas no paramétricas como se muestra en la figura 2.

Variables independientes: estímulo focal bajo gasto cardiaco. Estímulo contextual intervención de enfermería.

Variables dependientes: tensión arterial sistólica, frecuencia cardiaca, llenado capilar.

\section{ANÁLISIS ESTADÍSTICO DE DATOS}

Cuantificación

de la respuesta

adaptativa al estímulo

focal: bajo gasto caridaco
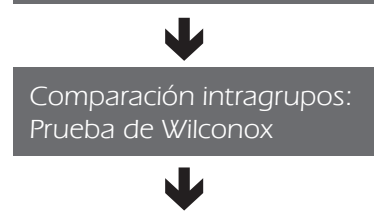

Comparación intragrupos:

Prueba de Kruskal-Wallis

Figura 2. Análisis estadístico

Fuente: producto de la investigación Respuesta adaptativa en el modo fisiológico del lactante con bajo gasto cardiaco en estado crítico, a las intervenciones cotidianas de enfermería en la unidad de cuidado intensivo pediátrico.

Cuantificación de la

respuesta adaptativa efectiva e inefectiva

al estímulo focal contextu

Tabla de contingencia:

Prueba de chi cuafrado 


\section{Resultados: análisis y discusión}

Para la construcción de los indicadores de adaptación positiva se definieron cuatro grupos de edad, dada la variabilidad de los signos vitales. Dicha agrupación se mantiene para el análisis estadístico. El 49,3\% de los pacientes tenía entre 2 y 6 seis meses de edad, y fue el grupo más representativo. La media para la edad fue de 7 meses.

\section{Diagnóstico clínico}

Además de bajo gasto cardiaco, los 75 pacientes tenían neumonía como diagnóstico clínico de base, en un 53,3\% de los casos, de los cuales el $60 \%$ se debió a virus sincitial respiratorio. La cirugía cardiovascular representó el 25,3\% de los casos, y fue el cierre de la comunicación interventricular la intervención quirúrgica más frecuente $(36,8 \%)$. E1 21,4\% restante se debió a sepsis, trauma craneoencefálico y cirugía general. El elevado porcentaje de pacientes con neumonía se explica por el hecho de que la investigación fue realizada durante los meses de pico epidemiológico para infecciones respiratorias en la ciudad de Bogotá.

\section{Inotrópicos y vasoactivos}

El corazón del lactante presenta una sensibilidad disminuida a las catecolaminas, por lo que son necesarias: primero, dosis cada vez más elevadas, y segundo, una terapia combinada. Aquellos pacientes que requieren tres o más medicamentos inotrópicos se encuentran en condición verdaderamente crítica (14). El $81 \%$ de los pacientes recibía dopamina, que era el medicamento más utilizado, seguido de la nitroglicerina (43\%) y la noradrenalina $(41 \%)$. Recordemos que algunos pacientes tenían más de un medicamento a la vez, por lo que los porcentajes deben tomarse de forma independiente. El levosimendano y la dobutamina fueron los medicamentos menos utilizados (3\%). Otros medicamentos administrados fueron adrenalina, vasopresina y milrinone.

La dosis específica de cada uno de los medicamentos no fue tenida en cuenta, dado que el criterio de severidad de bajo gasto cardiaco se estableció - como se indica en la metodología - de acuerdo con la cantidad de inotrópicos y vasoactivos que de forma simultánea y en dosis terapéuticas estuvieran recibiendo los lactantes (los goteos en dosis subterapéuticas por fase de destete fueron descartados).

\section{Duración de la intervención}

La duración promedio de la intervención cambio de posición fue de 17,13 minutos. La variabilidad del tiempo fue escasa en la mayoría de los pacientes, lo que otorga homogeneidad en la duración del estímulo generado. 


\section{Intervención de enfermeria: cambio de posición}

El cambio de posición cada dos horas es una intervención de enfermería de práctica común con los pacientes hospitalizados y es considerada de gran impacto en la prevención de zonas de presión y úlceras de piel $(15,16)$; sin embargo, el riesgo beneficio en los pacientes críticos debe ser tenido en cuenta y analizado con mayor detenimiento.

En los pacientes críticos, algunos estímulos (cambios de posición, por ejemplo) pueden desencadenar respuestas diversas de origen cardiovascular y otras pulmonares relacionadas (17); pero no se conoce con exactitud cuáles intervenciones de enfermería desencadenan mayor inestabilidad hemodinámica y cuáles son bien toleradas. En términos de adaptación, es posible entender la respuesta hemodinámica a dichas intervenciones de enfermería como una adaptación inefectiva si el resultado es inestabilidad o adaptación efectiva si, por el contrario, el paciente permanece estable tras la intervención de enfermería, siendo este último el fin deseable.

\section{Tensión arterial sistólica}

Comparación intragrupos: al comparar las cifras de tensión arterial sistólica de los sujetos dentro de cada grupo se observa que estas son significativamente diferentes al minuto 30 con respecto al minuto 0 en todos los grupos.

Prueba de Wilcoxon: grupo I $(p=0,000)$, grupo II $(p=0,04)$ y grupo III $(\mathrm{p}=0,02)$.

Comparación intergrupos: así mismo, al realizar la comparación intergrupos (I, II y III) se observa diferencia de los valores de tensión arterial sistólica de un grupo a otro en cada medición. Prueba de Kruskal-Wallis: minuto $5(\mathrm{p}=0,000)$, minuto $15(\mathrm{p}=0,02)$ y minuto $30(\mathrm{p}=0,000)$.

Como se observa en la figura 3 , pasados 30 minutos del cambio de posición un porcentaje significativo de lactantes se encuentra en adaptación inefectiva, dada por hipertensión e hipotensión. La capacidad de adaptación en el modo fisiológico se ve seriamente comprometida en el grupo III, donde el $64 \%$ de los lactantes continúa en adaptación inefectiva, dada principalmente por hipotensión, y solo el 36\% alcanza la adaptación efectiva.

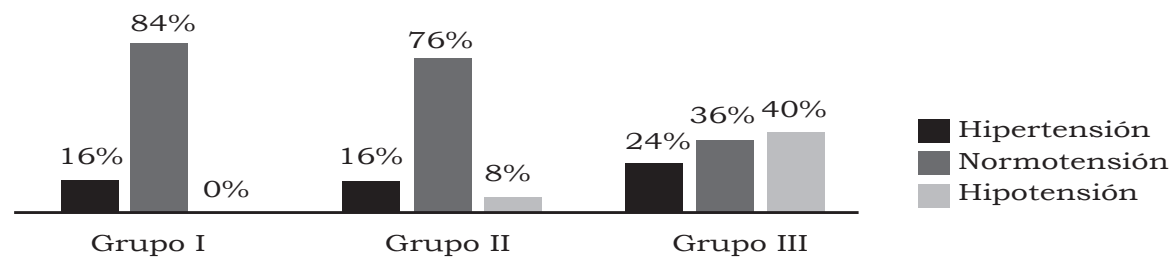

Figura 3. Tensión arterial sistólica al minuto 30 del cambio de posición

Fuente: producto de la investigación Respuesta adaptativa en el modo fisiológico del lactante con bajo gasto cardiaco en estado critico, a las intervenciones cotidianas de enfermería en la unidad de cuidado intensivo pediátrico. 
Estos hallazgos se contraponen a los resultados de diversos estudios que concluyen que los cambios en las constantes vitales de los pacientes a quienes se les realiza cambio de posición son respuestas sutiles, dadas por cambios transitorios que luego de 5 o 10 minutos retornan a sus valores iniciales (18-21). Cabe aclarar que dichos estudios han sido realizados solo en adultos y que los pacientes hemodinámicamente inestables han sido excluidos. A la fecha de realización de esta investigación no se encontraron estudios en pediatría con pacientes críticos que fueran comparables. Además, observando la figura 3 se identifica que cada grupo de bajo gasto cardiaco responde de manera diferente frente al estímulo cambio de posición (Kruskal-Wallis $\mathrm{p}=0,000$ al minuto 30) y que la respuesta adaptativa depende de la severidad del bajo gasto cardiaco. A mayor severidad del bajo gasto cardiaco, mayor presencia de adaptación inefectiva (prueba de independencia con chi cuadrado $\left.\left[\chi^{2}\right]: p=0,00\right)$.

\section{Frecuencia cardiaca}

Comparación intragrupos: al comparar las cifras de frecuencia cardiaca de los sujetos dentro de cada grupo, se observa que estas son significativamente diferentes al minuto 30 con respecto al minuto 0 .

Prueba de Wilcoxon: grupo I $(p=0,000)$, grupo II $(p=0,000)$ y grupo III $(\mathrm{p}=0,000)$.

Comparación intergrupos: así mismo, al realizar la comparación intergrupos (I, II y III), se observa diferencia de los valores de frecuencia cardiaca de un grupo a otro en cada medición, pero que es estadísticamente significativa en los minutos 15 y 30. Prueba de Kruskal-Wallis: minuto 5 $(\mathrm{p}=0,116)$, minuto $15(\mathrm{p}=0,016)$ y minuto $30(\mathrm{p}=0,039)$.

Los tres grupos de bajo gasto cardiaco mostraron cambios estadísticamente significativos en las mediciones al minuto 5, 15 y 30, en comparación con el minuto 0 . Dichos cambios perduran aun al minuto 30 como se observa en la figura 4, especialmente en los grupos II y III.

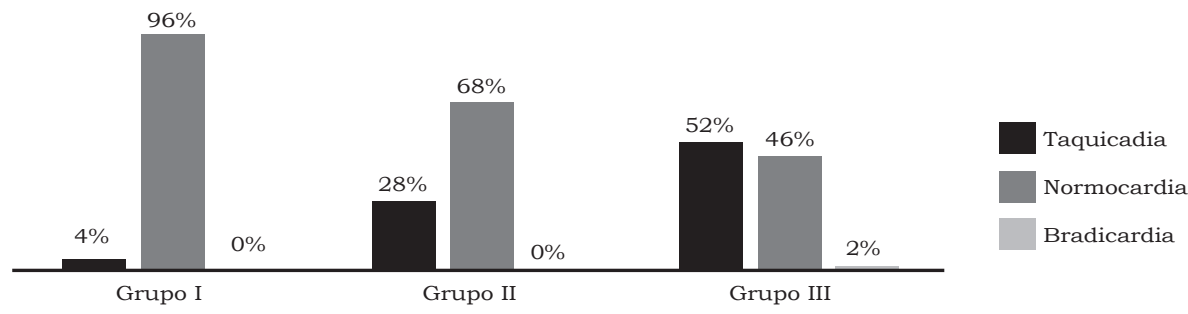

Figura 4. Frecuencia cardiaca al minuto 30 del cambio de posición

FUENTE: producto de la investigación Respuesta adaptativa en el modo fisiológico

126 del lactante con bajo gasto cardiaco en estado crítico, a las intervenciones cotidianas de enfermería en la unidad de cuidado intensivo pediátrico. 
Este hallazgo difiere de las conclusiones presentadas en un estudio prospectivo, donde se reporta que tras el cambio lateral de posición, las variaciones en la frecuencia cardiaca son mínimas, no perduran en el tiempo y no exceden los límites de normalidad (22). Por el contrario, en la presente investigación no solo se observa que los cambios en la frecuencia cardiaca de los lactantes perduran incluso después del minuto 30, sino que superan los limites de normalidad para la edad y consolidan estados de adaptación inefectiva al estímulo contextual cambio de posición.

\section{Llenado capilar}

Comparación intragrupos: al comparar el llenado capilar de los sujetos dentro de cada grupo, se observa que son significativamente diferentes al minuto 30 con respecto al minuto 0, en los grupos II y III. Prueba de Wilcoxon: grupo I ( $p=0,778)$, grupo II $(p=0,017)$ y grupo III $(p=0,012)$.

Comparación intergrupos: la diferencia en el llenado capilar de un grupo a otro no es estadisticamente significativa en ninguna de las mediciones. Prueba de Kruskal-Wallis: minuto $5(\mathrm{p}=0,073)$, minuto $15(\mathrm{p}=$ $0,432)$ y minuto $30(\mathrm{p}=0,083)$.

En la figura 5 se observa que al minuto 30 el $46 \%$ de los niños del grupo III permanece con el llenado capilar disminuido. Por otra parte, todos o la mayoría de los niños de los grupos I y II presentan un llenado capilar normal.

Al analizar en conjunto los tres indicadores de bajo gasto cardiaco (tensión arterial sistólica, frecuencia cardiaca y llenado capilar) con los resultados de las mediciones repetidas en la intervención cambio de posición, es posible identificar que la primera respuesta fisiológica frente al shock es la elevación de la frecuencia cardiaca por encima de los valores normales para la edad. Ello consolida la respuesta adaptativa inefectiva: taquicardia, identificada en esta investigación y presente en los tres grupos de bajo gasto cardiaco desde la primera medición al minuto 5. Aun al minuto 30 perduró en los siguientes porcentajes: GI (grupo I) = 4\%, GII = $28 \%$, GIII $=52 \%$ (figura 4 ).

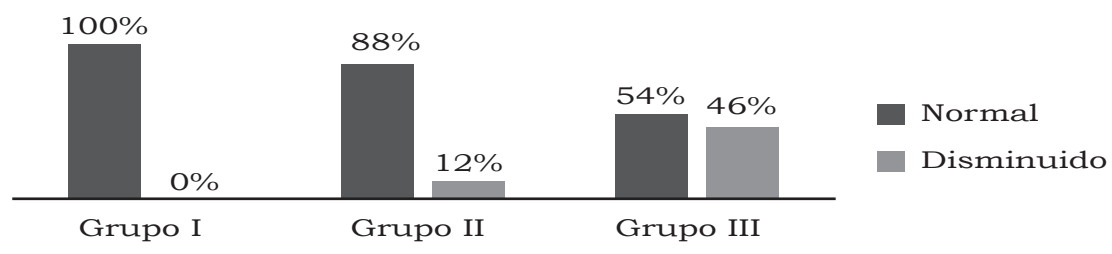

Figura 5. Llenado capilar al minuto 30 del cambio de posición

Fuente: producto de la investigación Respuesta adaptativa en el modo fisiológico del lactante con bajo gasto cardiaco en estado crítico, a las intervenciones cotidianas de enfermería en la unidad de cuidado intensivo pediátrico. 
La elevación de la frecuencia cardiaca puede, por sí sola, mantener el gasto cardiaco y evitar la hipotensión, dado que mientras en los adultos el gasto cardiaco depende mayormente de la fuerza de contracción del corazón, en los niños depende más de la frecuencia del latido. Sin embargo, no siempre es suficiente y, en general, se agota con el tiempo.

Un segundo mecanismo compensatorio es la concentración del flujo sanguíneo para mantener cifras tensionales que permitan la perfusión constante y suficiente de los órganos vitales. Para lograr aumentar la presión de perfusión central es necesario disminuir el flujo sanguíneo de órganos secundarios como la piel y en casos más severos se ve también disminuida la circulación esplácnica y renal. El indicador de adaptación positiva llenado capilar evalúa de forma confiable la perfusión de la piel. Los resultados en esta investigación al minuto 30 fueron los siguientes: GI $=0 \%, \mathrm{GII}=12 \%, \mathrm{GIII}=46 \%$ (figura 5 ). Estos valores evidencian la puesta en marcha del mecanismo compensatorio antes mencionado.

Cuando aparece la respuesta adaptativa inefectiva hipotensión, se puede considerar que los mecanismos compensatorios iniciales se han agotado o fueron insuficientes. La hipotensión se presentó en la siguiente proporción: $\mathrm{GI}=0 \%, \mathrm{GII}=8 \%$ y GIII $=40 \%$ al minuto 30 (figura 3 ). Sin embargo, puede apreciarse que el porcentaje de lactantes con hipotensión es más bajo que el porcentaje de lactantes con taquicardia y llenado capilar disminuido, lo que evidencia el éxito de los mecanismos compensatorios en el síndrome de bajo gasto cardiaco. También los fármacos vasoactivos, inotrópicos y cronotrópicos que reciben los pacientes en infusión continua desempeñan un papel fundamental al potencializar y prolongar la respuesta fisiológica adaptativa.

Es muy importante destacar que en el grupo III persiste la hipotensión pasados 30 minutos en un alto porcentaje (40\%), lo que demuestra la fragilidad hemodinámica del paciente crítico y comprueba que una intervención de enfermería tan común, como el cambio de posición, altera el equilibrio fisiológico, que puede resultar muy difícil de restablecer.

La severa respuesta adaptativa inefectiva que se observa en el grupo III se relaciona con los resultados de un estudio experimental hecho en ratones con disfunción miocárdica inducida y un grupo control con corazón sano, a los cuales se les cambió de posición repetidamente. E1 estudio concluye que el volumen sistólico ventricular se reduce significativamente en el corazón enfermo con cada cambio de posición, mientras que el corazón sano mantiene un volumen sistólico normal en todas las posiciones (23). La severidad de la insuficiencia miocárdica que se genera en el sindrome de bajo gasto cardiaco, sin lugar a dudas, explica la intolerancia a la intervención de enfermeria: cambio de posición particularmente en los grupos II y III.

Otro aspecto que llama la atención en esta investigación con relación a los cambios de posición es el ángulo de la cabecera de la cama. En los datos derivados de la observación se evidencia que para realizar el 
cambio de posición del lactante, el personal de enfermería lleva el ángulo de la cabecera a $0^{\circ}$ por un tiempo promedio de 17,13 minutos, y una vez finalizada la intervención, el lactante es devuelto de manera súbita al ángulo inicial de $45^{\circ}$. Se debe tener en cuenta que los lactantes se encuentran en la unidad de cuidado intensivo pediátrico, donde todas las camas son de tamaño estándar para adulto, dado que se admiten pacientes de 2 meses a 17 años de edad. Esto hace que en el ángulo de $45^{\circ}$ todo el cuerpo del lactante quede en posición oblicua y los cambios hemodinámicos mencionados se acentúen.

Son necesarios estudios acerca de los efectos tanto benéficos como potencialmente adversos de las intervenciones cotidianas de enfermería, porque en su mayoría se desconocen en el paciente crítico, no solo en el ámbito pediátrico, sino también en los pacientes adultos, dado que los estudios existentes son excluyentes. Por otra parte, se requiere continuar con la construcción de indicadores de adaptación positiva específicos para los diferentes grupos de pacientes críticos; de esta manera, enfermería gana herramientas útiles para la práctica clinica que se fundamenta en su propio conocimiento disciplinar.

En un estudio descriptivo se encontró que las enfermeras clínicas usan más la práctica del conocimiento prestado de otras ciencias de la salud que del conocimiento que enfermería como disciplina les ofrece, ya que lo consideran confuso y poco aplicable en el cuidado del paciente crítico (24). Con esta investigación se demuestra que es posible utilizar el conocimiento teórico propio de enfermería para resolver problemas de la práctica clínica cotidiana.

La intervención de enfermería cambio de posición debe realizarse con extrema precaución en los lactantes con bajo gasto cardiaco que reciban dos o más inotrópicos o vasoactivos en infusión continua. Producto de esta investigación se recomienda para el cuidado de la piel:

1. Intentar el cambio de posición sin modificar el ángulo de la cama $\mathrm{y}$, de ser indispensable, hacerlo de forma progresiva observando la respuesta hemodinámica.

2. La cabecera a $0^{\circ}$ mejora la hemodinámica del lactante; sin embargo, se recomienda mantener el ángulo de $45^{\circ}$ por los efectos positivos en la mecánica ventilatoria y la prevención del reflujo gástrico y la broncoaspiración.

3. Con la ayuda de rollos de soporte, realizar el cambio de posición de forma gradual hasta obtener la posición deseada y detenerse en presencia de hipotensión, hasta que esta haya desaparecido.

4. Emplear guantes o globos de látex llenos de agua para proteger las prominencias óseas.

5. Considerar espaciar a más de dos horas el cambio de posición, en presencia de inestabilidad hemodinámica secundaria y acorde a la efectividad de las medidas alternativas instauradas para la protección de la integridad de la piel. 


\section{Conclusiones}

La respuesta adaptativa en el modo fisiológico en lactantes de 2 a 23 meses de edad depende de la severidad del bajo gasto cardiaco: a mayor severidad del bajo gasto cardiaco, mayor presencia de adaptación inefectiva. Es indispensable para la enfermera conocer la respuesta adaptativa en el modo fisiológico del paciente pediátrico en estado crítico para comprender y predecir con mayor precisión la respuesta a las intervenciones de cuidado que planee para este grupo de pacientes.

Es posible la cuantificación de la capacidad de adaptación de un sistema humano a través de indicadores sensibles y específicos de adaptación positiva. Este es un aporte teórico de gran valor para el modelo de adaptación de Roy, pero también constituye un aporte significativo para la práctica clínica de enfermería en las unidades de cuidado intensivo, porque permite medir la tolerancia fisiológica a las intervenciones cotidianas de enfermería.

\section{Recomendaciones}

La enfermera clínica necesita hacer un uso sistemático del conocimiento disciplinar y de los resultados de investigaciones que, como esta, aportan evidencia científica a la toma autónoma de decisiones en la práctica que no obedezcan solo al seguimiento rígido de guías y protocolos estandarizados, sino que respondan a las necesidades de cuidado individualizado del paciente en estado crítico.

Los estudios experimentales y casi experimentales encuentran una profunda limitación en el ámbito del cuidado intensivo, por lo que estudios de tipo observacional descriptivo son una excelente alternativa. Los datos obtenidos en los estudios no experimentales son igualmente valiosos y, sin duda, con un análisis estadístico riguroso se obtendrán resultados de alta confiabilidad.

\section{Referencias}

1. Roth S, Adatia I, Pearson G. Summary proceedings from the cardiology group on postoperative cardiac dysfunction. Pediatrics. 2006;117(3):40-6.

2. Calderón J, Patiño E. Insuficiencia cardiaca en la edad pediátrica. En: Fause A, Calderón J, Zabal C, Buendía A. Cardiología pediátrica. México: Editorial Médica Panamericana; 2013. p. 473.

3. Roy C, Andrews H. The Roy adaptation model. 2nd ed. Stamford: Appleton \& Lange; 1999.

4. Ramos J, Guáqueta S. Respuesta adaptativa en el modo fisiológico, del lactante con bajo gasto cardiaco en estado crítico, a las intervenciones cotidianas de enfermería en la UCIP. Tesis de maestría, Universidad Nacional de Colombia; 2011 [citado 16 feb 2014]. Disponible en: http://www.bdigital.unal.edu.co/6349/1/539691.2012.pdf. 
5. Maya LC. Disfunción miocárdica en pediatría. En: Pinto A, Maya LC, Rivero E, editores. Principios en la atención del niño crítico. Bogotá: Distribuna; 2004. p. 61-75.

6. Grupo de Estudio para el Desarrollo del Modelo de Adaptación. Análisis de los conceptos del modelo de adaptación de Callista Roy. Aquichan. 2002;2(002):19-23.

7. Massé L, Antonacci M. Low cardiac output syndrome: identification and management. Crit Care Nur Clin North Am. 2005;17(4):375-83.

8. Burns N, Grove S. Investigación en enfermería. 3a ed. Madrid: E1seiver; 2005.

9. Polit D, Hungler B. Diseños de investigación para estudios cuantitativos. En: Investigación científica en ciencias de la salud. 6a ed. México: McGraw-Hill; 2000. p. 191.

10. Arango G. Los comités de ética de la investigación: objetivos, funcionamiento y principios que buscan proteger. Investig Enferm. Imagen Desarr. 2008;10(1):9-20.

11. Concejo de Organizaciones Internacionales de las Ciencias Médicas. Colaboración con la Organización Mundial de la Salud (OMS). Pautas éticas internacionales para la investigación biomédica en seres humanos [internet]. Ginebra; 2002, p. 5-89. Disponible en: http:// www.paho.org/Spanish/BIO/CIOMS.pdf.

12. Resolución 008430/1993 de 4 de octubre, por la cual se establecen las normas científicas, técnicas y administrativas para la investigación en salud. Ministerio de Salud de la República de Colombia.

13. Duffau G. Tamaño muestral en estudios biomédicos. Rev Chil Pediatr. 2009;70(4):314-24.

14. Patiño E, Juanico A. Combinación inotrópica optima en pediatría. En: Verdejo J, Patiño E, Nava S. Cardiopatías congénitas. México: Pydesa; 2013.

15. Bours G, Laat E, Halfens R, Lubbers M. Prevalence, risk factors and prevention of pressure ulcers in Dutch intensive care units. Results of a cross-sectional survey. Intensive Care Med. 2001;27(10):1599-1605.

16. Shahin E, Dassen T, Halfens R. Pressure ulcer prevalence in intensive care patients: a cross-sectional study. J Eval Clin Pract. 2008;14(4):563-8.

17. Marklew A. Body positioning and its effect on oxygenation a literature review. Nurs Care Crit. 2006;11(1):16-22.

18. Banasik J, Emerson R. Effect of lateral positions on tissue oxygenation in the critically ill. Heart Lung. 2001;30(4):269-76.

19. Jones A, Dean E. Body position change and its effect on hemodynamic and metabolic status. Heart Lung. 2004;33(5):281-90.

20. Gawlinski A, Dracup K. Effect of positioning on SvO2 in the critically ill patient with a low ejection fraction. Nurs Res. 1998;47(5):293-9.

21. Laat E, Schoonhoven L, Grypdonck M, Verbeek A, Ruurd G, Pickkers P. Early postoperative 30o lateral positioning after coronary artery surgery: influence on cardiac output. J Clin Nursing. 2007;16(4):654-61. 
22. Thomas P, Paratz J, Lipman J, Lipman J, Stanton W. Lateral positioning of ventilated intensive care patients: A study of oxygenation, respiratory mechanics, hemodynamics, and adverse events. Heart Lung. 2007;36(4):277-86.

23. Siepe M, Ruegg D, Noelle M, Giraud J, Carrel T, Tevaearai H. Effect of acute body positional changes on the hemodynamics of rats with and without myocardial infarction. Exp Physiol. 2005;90(4):627-34.

24. Gallego M, Leal M, Ramos X. Elementos teóricos del cuidado de enfermería en la unidad de cuidado intensivo. Repert Med Cir. 2009;18(2):76-8. 